\title{
Generation of pure spin currents in a single electron transistor with a superconducting island
}

\author{
Marius V. Costache ${ }^{\mathrm{a}}$, and Sergio O. Valenzuela*a,b,c \\ ${ }^{a}$ Catalan Institute of Nanotechnology (CIN2-ICN), Bellaterra E-08193, Spain \\ ${ }^{\mathrm{b}}$ Institució Catalana de Recerca i Estudis Avançats (ICREA), Barcelona E-08010, Spain \\ ${ }^{\mathrm{c}}$ Physics Department, Universitat Autónoma de Barcelona, Bellaterra E-08193, Spain
}

\begin{abstract}
We describe a spin filter at the single-electron level that produces pure spin currents with no net bias. Our device is based on the ground-state energetics of a single-electron transistor comprising a superconducting island connected to normal leads via tunnel barriers with different resistances that break spatial symmetry. The current has opposite spin polarization when the current is reversed, which leads to a dc spin current when applying an alternating charge current with zero mean, as expected in a spin ratchet. We demonstrate spin transport and quantify the spin ratchet efficiency by means of ferromagnetic leads with known spin polarization.
\end{abstract}

Keywords: Spin current, single electron transistor, spin filter, spin ratchet.

\section{INTRODUCTION}

During the last two decades, there has been a renewed interest in the research of electron spin physics by electrical means in the solid state community, yielding a variety of spectacular phenomena ${ }^{1}$. The interest is motivated by the quest to understand basic physical principles underlying the electron and nuclear spin interactions and by possible technological applications. The use of spins to encode information promises the integration of logic computation and storage whereas, in coherent systems, it can lead to methods for computation that would be robust against environmental decoherence $^{2}$. A key element to attain these goals is the development of spin current generators. Traditionally, this has been achieved by means of ferromagnetic materials. There, currents are naturally spin polarized due to the different density of states and associated mobilities of spin-up and spin-down electrons. An unpolarized current entering a magnetic material will become polarized via spin-flip processes. However, more recently, spin filters based on quantum $\operatorname{dots}^{3-5}$ and a variety of spin pumps ${ }^{6-8}$ and ratchets ${ }^{9-12}$ have also been proposed.

The concept of spin ratchets is attracting increased attention. In solid state, a ratchet usually refers to directed transport in the presence of a signal or perturbation that drives the system without an obvious bias in any preferred direction of motion $^{13,14}$. The perturbation generates useful work, for instance the transport of particles, when combined with asymmetry, often realized by a so-called ratchet potential (Fig. 1). Experimental realizations of ratchets are spread over many different fields of biology, chemistry and physics where the perturbation may be external to the system (e.g. induced by an experimentalist) or intrinsic to it (e.g. non-thermal noise). In mesoscopic structures, experiments have demonstrated ratchets in both the quantum and classical limits for charge ${ }^{15,16}$ and superconducting vortex ${ }^{17-21}$ motion and in SQUIDs ${ }^{22}$ (superconducting quantum interference devices). On such small scales, noise rectification with ratchets can be used to control particle transport and has become one of the most promising techniques for powering nanodevices.

A variety of ratchets have been proposed in pursuit of unidirectional spin currents and spin control ${ }^{9-12}$. A pure spin ratchet generalizes the particle ratchet mechanism, enabling pure spin currents by means of broken spatial symmetry. Thus, an indispensable hallmark for a spin ratchet is the breaking of the inversion symmetry for spin but not charge, whereby the ratchet-potential easy direction for one spin orientation is opposite to the ratchet-potential easy direction for the other spin orientation (Fig. 1). Recent theoretical efforts employ mesoscopic semiconductors and non-uniform magnetic fields ${ }^{9}$, asymmetric periodic structures with Rashba spin-orbit interaction ${ }^{10}$, and double-well structures combined with local external magnetic fields and resonant tunneling ${ }^{12}$.

*SOV@icrea.cat; phone 3493586 8305; fax 3493586 8313; www.icn.cat/pend

Spintronics IV, edited by Henri-Jean M. Drouhin, Jean-Eric Wegrowe, Manijeh Razeghi,

Proc. of SPIE Vol. 8100, 81000I · @ 2011 SPIE · CCC code: 0277-786X/11/\$18 - doi: 10.1117/12.890231 


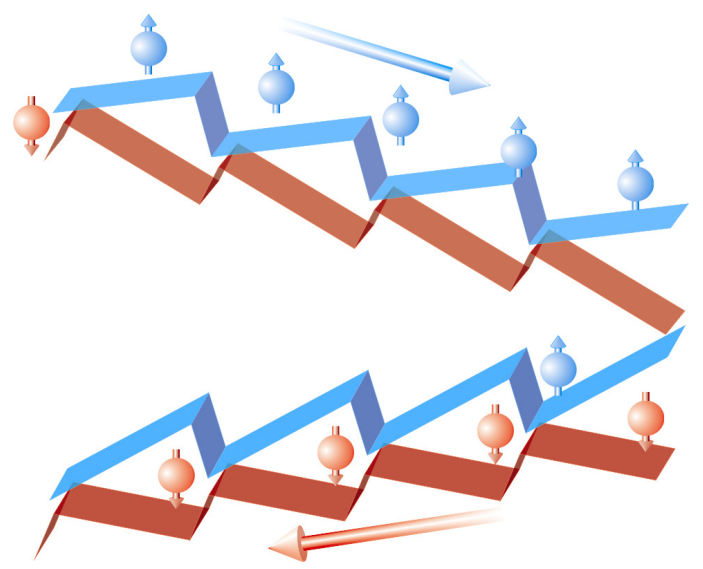

Figure 1. Schematic of the potential in a rocking spin ratchet. The potential is reversed along the motion direction for spins with opposite orientation. The asymmetry leads to easy motion of spin-up electrons to the right and spindown electrons to the left.

Our spin-filter is different from what has been proposed before and fulfills the above definition of a spin ratchet ${ }^{23}$. A small-volume superconducting (S) island is connected via tunnel junctions with two normal metal electrodes [N(l) and $\mathrm{N}(\mathrm{r})$ ] to form an asymmetric NSN single electron transistor (SET) with different tunneling resistances $R_{1, \mathrm{r}}$ [Fig. 2 (a)]. As we will describe in the following section, the main requirements for the spin ratchet effect to be observed are rather simple: i) a small-volume thin superconducting island, ii) a Zeeman-induced splitting imposed by an applied magnetic field, and iii) an asymmetric tunneling to the metal electrodes. The first condition results in quasiparticle-dominated transport in the subgap transport region of the superconducting island, the second in spin filtering, and the third in the needed spatial asymmetry to obtain a net spin transport when an ac charge current is applied.

\section{DEVICE CONCEPT}

\subsection{Tunneling rates and volume of the superconducting island}

At low temperatures, parity effects in the superconducting island are important ${ }^{24-27}$. When the number of (excess) conduction electrons $n$ is odd, there is necessarily one unpaired electron that is manifest as a quasiparticle excitation. The ground state energy of the system for odd $n$ is higher than for even $n$ by the superconducting gap $\Delta$, which in our design is larger than the charging energy, $E_{\mathrm{c}}$. The system energy diagram is shown in Fig. 2 (b). Each parabola corresponds to a specific value of $n$. A voltage on a gate $V_{\mathrm{g}}$ sets the induced gate charge $Q=V_{\mathrm{g}} C_{\mathrm{g}}$ on the island, with $C_{\mathrm{g}}$ the capacitive coupling between the island and the gate.

A bias voltage $V$ applied across the SET drives the system. At low $V$, and because the odd states have a large energy, two-electron transfers between $\mathrm{N}$ and $\mathrm{S}$ tend to be important, as observed historically ${ }^{25-27}$. Andreev reflection is a mechanism supporting such transfer with rates $\Gamma_{1, \mathrm{r}}^{\mathrm{A}}$ that are inversely proportional to the square of the tunneling resistances $R_{1, \mathrm{r}}$ of the junctions ${ }^{28}$. At high enough $V$, it becomes energetically favorable to introduce a single quasiparticle in the island. While this particle stays in the island, it blocks the Andreev cycle since any two electron tunneling process becomes highly unfavorable in this situation ${ }^{25-29}$. Due to the slow escape rate of any specific quasiparticle, $\Gamma_{1, \mathrm{r}}^{\mathrm{o}}$, from odd (o) to even (e) states, the current drops at the quasiparticle trapping thresholds. This has been observed in a number of classical experiments. ${ }^{27,29}$

In order to transfer spin currents through the NSN SET with large efficiency, it is necessary to enhance the quasiparticle transport and suppress the Andreev cycle, which transfers pairs of spin-up/down electrons and thus it does not transport spins. It is possible to accomplish this, to a certain extent, by increasing $R_{1, \mathrm{r}}$. Andreev reflections depend on the precise geometry near the junctions as well as on impurities and scattering sites but they are second-order processes that are suppressed in junctions with low enough transparency ${ }^{30-33}$. On the other hand, the rates $\Gamma_{1, \mathrm{r}}^{\mathrm{oe}}$ and $\Gamma_{1, \mathrm{r}}^{\mathrm{eo}}$ are first order processes that depend less dramatically on the junction transparencies. Therefore, the spin polarization of current through 
the SET should increase when $R_{\mathrm{l}, \mathrm{r}}$ increase. However, the increase of $R_{\mathrm{l}, \mathrm{r}}$ cannot be too large because the magnitude of the spin current will decrease and could become undetectable or too small for any practical use.

An additional enhancement of the quasiparticle transport can be accomplished when noting that it is regulated by the escape rates, $\Gamma_{1, \mathrm{r}}^{\mathrm{oe}}$. This is because the odd-even rates $\Gamma_{1, \mathrm{r}}^{\mathrm{oe}}$ are usually much smaller than $\Gamma_{1, \mathrm{r}}^{\mathrm{eo}}$ and given by ${ }^{28}$ :

$$
\Gamma_{1, \mathrm{r}}^{\mathrm{e}}=\left(2 e^{2} R_{\mathrm{l}, \mathrm{r}} \rho_{\mathrm{n}} V_{\mathrm{S}}\right)^{-1} \text {, }
$$

where $e$ is the electron charge, $\rho_{\mathrm{n}}$ is the normal density of states of the superconductor per unit volume (including spin), and $V_{\mathrm{S}}$ is the volume of the superconducting island. The reason why $\Gamma_{1, \mathrm{r}}^{\mathrm{oe}}<<\Gamma_{1, \mathrm{r}}^{\mathrm{e}}$ is because in the former a specific quasiparticle must be removed from the superconducting island whereas in the latter all of the quasiparticle states are involved ${ }^{28}$.

Equation (1) implies that, for a given $R_{\mathrm{l}, \mathrm{r}}$, the escape rate increases when the volume of the island decreases, which is due to the normalization of the wavefunction of an unpaired quasiparticle in the island. This observation results in the first condition mentioned in the introduction for the spin ratchet to work efficiently. Below we will determine the magnitude of the rates involved in our devices.

(a)
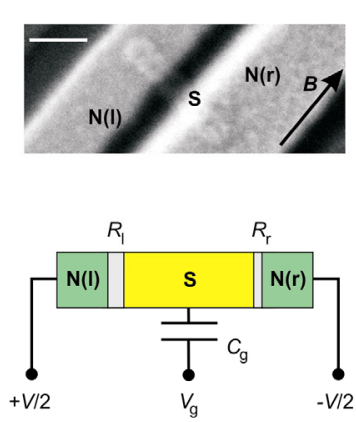

(b)

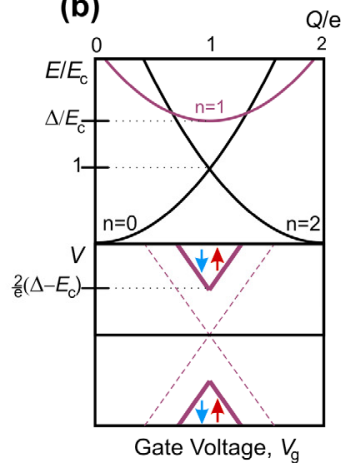

(c)

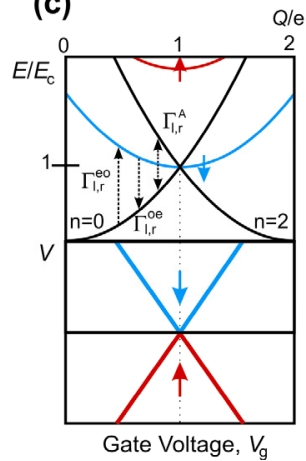

(d)

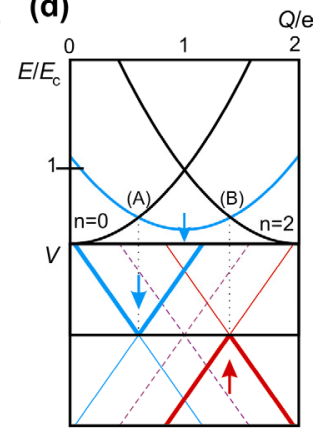

Figure 2. Single electron transistor (SET) spin ratchet. (a) Electron scanning microscope image of the SET. The bar is $100 \mathrm{~nm}$ long. (b)-(d), SET energetics of Cooper-pair and quasiparticle states (top) and associated below-gap voltage thresholds (bottom) for single and two-electron transport at low temperatures for $B=0(\mathrm{~b}), B=B_{\mathrm{SR}}$ (c) and $B>B_{\mathrm{SR}}(\mathrm{d})$. Dashed and solid lines represent the positions of the Andreev and quasiparticle conductance thresholds, respectively.

\subsection{Spin filtering and Zeeman-induced quasiparticle splitting}

In order to break the symmetry between spin-up and spin-down transport, a magnetic field $B$ is applied in-plane along the axis of the electrodes [spin up (down) refers to spins parallel (antiparallel) to $B$ ]. This field splits the quasiparticle levels (e.g. $n=1^{\downarrow}$ and $n=1$ ) by the Zeeman energy ${ }^{34} E_{\mathrm{Z}}=g \mu_{\mathrm{B}} B$, where $g$ is the $g$-factor of the superconductor and $\mu_{\mathrm{B}}$ the Bohr magneton, but it does not affect the Cooper-pair states (e.g. $n=0$ and $n=2)$, which are singlet states. The island thickness is chosen to be very small so that orbital-depairing is minimized and $B$ weakly reduces $\Delta$ [Fig. 2, (c) and (d)]. With increasing $B$, the $n=1^{\downarrow}$ state shifts down continuously and, at $B_{\mathrm{SR}}=2\left(\Delta-E_{\mathrm{c}}\right) /\left(g \mu_{\mathrm{B}} B\right)$ [Fig. 2 (c)], it becomes degenerate with both the zero $(n=0)$ and the one $(n=2)$ excess Cooper-pair states for $Q / e=1$.

Zeeman splitting favors the trapping of a quasiparticle in the island with spin down orientation $\left(n=1^{\downarrow}\right)$, which can be demonstrated straightforwardly. Based on the discussion in section 2.1, we consider $\Gamma_{1, \mathrm{r}}^{\mathrm{A}}<<\Gamma_{1, \mathrm{r}}^{\mathrm{oe}}<<\Gamma_{1, \mathrm{r}}^{\mathrm{e}}$. In this situation, when transitions to state $n=1^{\downarrow}$ become energetically favorable, they occur very fast, and the charge current in the SET is limited by the specific quasiparticle escape rates $\Gamma_{1, \mathrm{r}}^{\mathrm{oe}}$ and the average occupation of $n=1^{\downarrow}$ is thus $\Gamma_{1, \mathrm{r}}^{\mathrm{eo}} / \Gamma_{1, \mathrm{r}}^{\mathrm{eo}}$ $\left.+\Gamma_{1, \mathrm{r}}^{\mathrm{oe}}\right) \sim 1$.

\subsection{Asymmetric SET and spin currents}

Fig. 3 shows the relevant charge transport processes and their corresponding rates for a single electron transistor at $B_{\mathrm{SR}}$. The widths of the arrows represent the relative weight of the different rates. Fig. 3(a) concentrates on the relative rates magnitudes in general, whereas Fig. 3, (b) and (c), focus on the effect of different tunneling resistances in the $l$ and $r$ junctions. At low voltages and temperatures, only the states $n=0, n=2$ and $n=1^{\downarrow}$ are needed to describe the transport; 
low-probability cotunneling events to higher excited states can be disregarded, which is verified experimentally. Single electron tunneling processes in the $l$ and $r$ junctions cause transitions between even $(n=0,2)$ and odd in $n=1^{\downarrow}$ states with rates $\Gamma_{1}^{\mathrm{e}} ; \Gamma_{1}^{\mathrm{eo}}$ and $\Gamma_{\mathrm{r}}^{\mathrm{oe}} ; \Gamma_{\mathrm{r}}^{\mathrm{eo}}$, respectively, whereas two-electron Andreev processes cause transitions between even $n$ $=0$ and $n=2$ states with rates $\Gamma_{1, \mathrm{r}}^{\mathrm{A}}[$ Fig. 3 (a)].

(a)

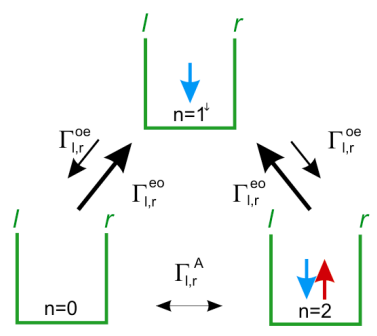

(b)

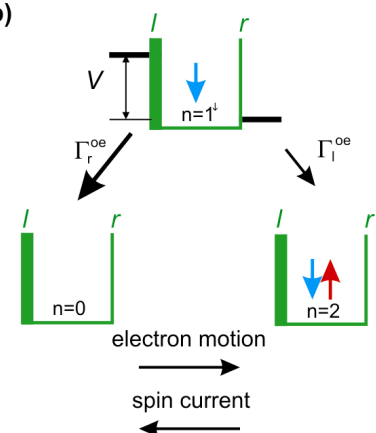

(c)

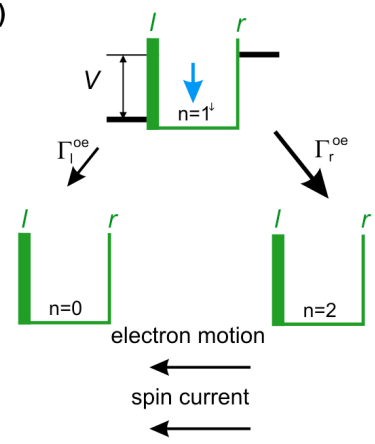

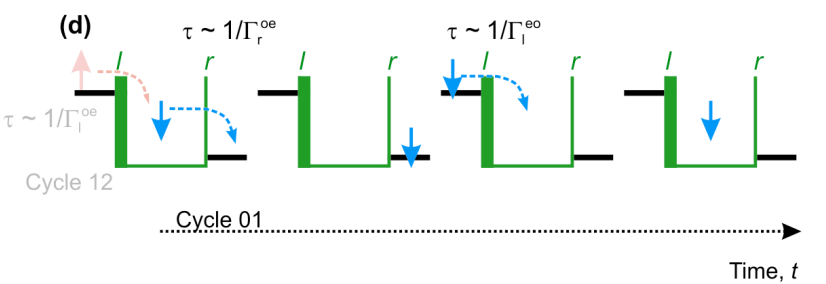

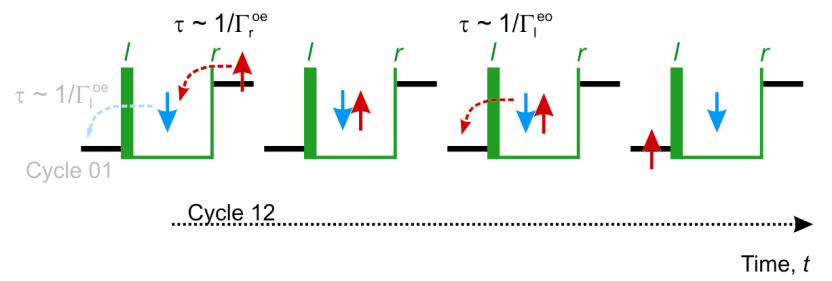

Figure 3. Illustration of the working principle of a single electron transistor (SET) spin ratchet; tunneling rates. (a), Schematic representation of the allowed charge transport processes. Each green box depicts the SET in the indicated state $\left(n=0, n=2\right.$, or $\left.n=1^{\downarrow}\right)$. The SET is designed such that $\Gamma_{1, \mathrm{r}}^{\mathrm{A}}<<\Gamma_{1, \mathrm{r}}^{\mathrm{e}}<<\Gamma_{1, \mathrm{r} \cdot}^{\mathrm{e}}$. The arrows widths represent the relative magnitude of the rates. (b) and (c), Dominant rates for positive and negative bias in the asymmetric SET at $B=B_{\mathrm{SR}}$. The thickness of the left and right lateral walls of the green boxes represents the transparency of the tunnel junctions. For electrons moving towards the right (b), the electron current is spin-down polarized [cycle 01 in (d)], whereas for electrons moving towards the left (c), the electron current is spin-up polarized [cycle 12 in (d)]. Overall, both processes contribute to a spin current with the same direction

A key point for our proposed spin-ratchet mechanism is that the ground state energetics of the SET dictates that different junction transparencies result in transport of spins with opposite orientation for positive and negative $V$. This explains the requirement that, for example, $\Gamma_{1}^{\mathrm{oe}}<\Gamma_{\mathrm{r}}^{\mathrm{oe}}$, where the $l$ junction transparency is arbitrarily chosen to be smaller than that of the $r$ junction. Fig. 3, (b) and (c), show the rates that dominate the transport of the asymmetric SET when electrons flow from left to right and from right to left, respectively. Because $\Gamma_{1}^{\mathrm{oe}}<\Gamma^{\mathrm{oe}}$, a quasiparticle removal process is more likely associated with a tunneling event in which either a quasiparticle directly tunnels off the island to the right lead [Fig. 3 (b) and (d)] or, for opposite bias, an electron from the right lead tunnels onto the island to form a Cooper-pair with an existing quasiparticle [Fig. 3 (c) and (d)]. Tunneling events through the low-transparency left-junction may occur but with smaller probability.

As a direct consequence, transport of electrons from left to right [Fig. 3 (b)] mostly involves the $n=1^{\downarrow}$ and $n=0$ states (cycle 01) because cycling between the $n=1^{\downarrow}$ and $n=2$ requires an electron tunneling from the left lead to remove the quasiparticle [Fig. 3 (d)]. In an analogous way, transport of electrons from right to left [Fig. 3 (c)] mostly involves the $n$ $=1^{\downarrow}$ and $n=2$ states (cycle 21) because cycling between the $n=1^{\downarrow}$ and $n=0$ requires the quasiparticle to tunnel off the island to the left lead [Fig. 3 (d)]. Therefore, for $\Gamma_{1}^{\mathrm{oe}}<\Gamma_{\text {r }}^{\mathrm{oe}}$, cycle 01 dominates at, say, positive $V$, while cycle 21 dominates at negative $V$.

Note that the effective easy direction of motion for one spin is thus opposite to the easy direction of motion for the other spin, as required in a spin ratchet (Fig. 1). Cycle 01 results in a spin-down polarized current for left-to-right electron motion, whereas cycle 21 results in spin-up polarized currents for right-to-left electron motion and overall both cases contribute to a spin current in the same direction. The efficiency to generate this spin current is directly related to the 
parameter $\alpha=\Gamma_{1}^{\mathrm{oe}} / \Gamma_{\mathrm{r}}^{\mathrm{oe}}$, which measures the asymmetry of the SET; the smaller $\alpha$, the more efficient is the spin ratchet. Because for opposite bias the rates involved are the same, the charge transferred is null in average when a voltage $V$ with zero mean (no net bias) is applied, thus the SET spin ratchet generates pure spin currents.

The spin-ratchet is realized at an applied magnetic field $B=B_{\mathrm{SR}}$. There, a finite $V$ will lead to spin motion. For $B>B_{\mathrm{SR}}$, the asymmetric SET acts as a diode that resolves spin [Fig. 1 (d)]. There, it is necessary to consider separately the degeneracies between $n=1^{\downarrow}$ and $n=0$ (A) and between $n=1^{\downarrow}$ and $n=2$ (B). In between the degeneracies, a single spindown quasiparticle stays in the island. Around the first degeneracy point (A), only cycle 01 can be involved in transport: a spin-down quasiparticle may tunnel onto and off the island resulting in a spin-down current. Around the second degeneracy point (B), only cycle 21 can be involved in transport: a spin-up quasiparticle tunnels onto the island to form a Cooper-pair with the spin-down quasiparticle, and subsequently a spin-up quasiparticle tunnels off, breaking a pair and leaving a spin-down quasiparticle behind; a sequence that results in a spin-up current.

\section{DEVICE FABRICATION AND CHARACTERIZATION MEASUREMENTS}

\subsection{SET fabrication}

Our SETs consist of a small-volume ( $6 \mathrm{~nm}$ thick by $40 \mathrm{~nm}$ wide by $250 \mathrm{~nm}$ long) aluminum (Al) superconducting island (S) connected to two nonsuperconducting electrodes, N(l) and N(r). Fig. 4 shows the main steps for their fabrication, which involve electron-beam lithography and multi-angle shadow evaporation to produce tunnel barriers in situ as described in our previous work ${ }^{35,36}$. A suspended shadow mask [Fig. 4 (a)] is first created on a highly-doped $\mathrm{Si}\langle 100\rangle$ wafer with thermally grown oxide. To this end, we use a methyl-methacrylate (MMA)/poly(methyl-methacrylate) (PMMA) bilayer in combination with selective electron-beam exposure. The base resist (MMA) has a sensitivity that is about 5 times larger than the top resist (PMMA), which allows us to generate a controlled undercut by exposing the bilayer with a dose that is sufficient to expose the MMA layer, but insufficient to expose the PMMA layer. The exposed bilayer is developed in an isopropanol / methyl-isobutyl-ketone solution and placed in a high-vacuum electron-beam evaporator (base pressure $<10^{-8}$ Torr).

The material evaporation sequence is shown in Fig. 4, (b) and (c). First, we evaporate Al perpendicular to the substrate (yellow), which creates the superconducting island. Next, the $\mathrm{Al}$ is oxidized in pure oxygen (100-150 mTorr for $40 \mathrm{~min}$ ) to generate insulating $\mathrm{Al}_{2} \mathrm{O}_{3}$ barriers. After the vacuum is recovered, the two electrodes, $\mathrm{N}(\mathrm{l})$ (blue) and $\mathrm{N}(\mathrm{r})$ (red), are sequentially deposited under angles of $50^{\circ}$ relative to the substrate normal, where the substrate is tilted in opposite direction for $\mathrm{N}(\mathrm{l})$ and $\mathrm{N}(\mathrm{r})$ [Fig. 4 (b)]. The sequential deposition leads to different tunneling resistances $R_{\mathrm{l}}$ and $R_{\mathrm{r}}$; the difference between $R_{1}$ and $R_{\mathrm{r}}$ can be enhanced by an additional oxidation step in between each lead deposition.

(a)

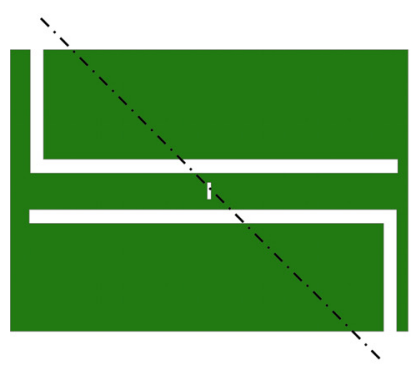

(b)

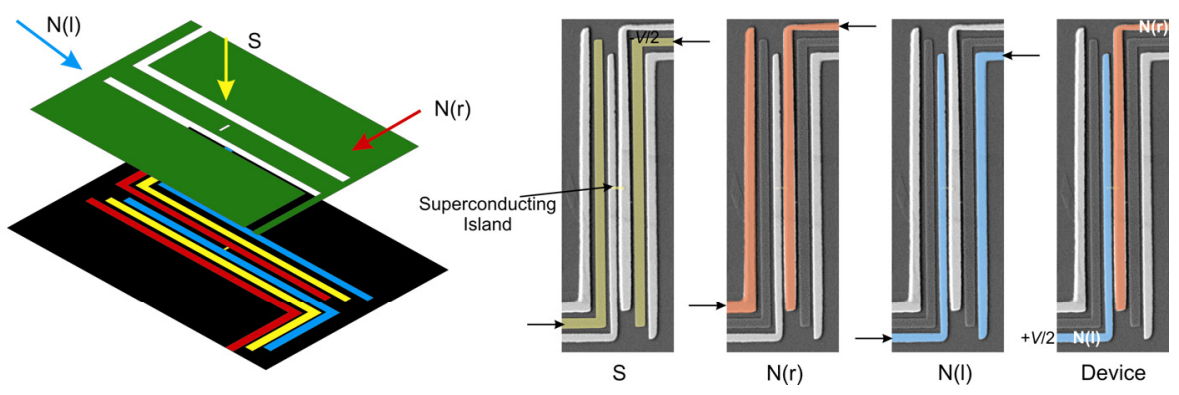

Figure 4. Sample fabrication. (a), Design of the suspended MMA/PMMA mask for shadow evaporation. The dashed line represents the rotation axis for shadow evaporation. (b), The device is fabricated by three sequential depositions as indicated by the arrows. Such a process results in a threefold projection of the mask. (c), Scanning electron microscope images of a device showing, from left to right, the deposition sequence of the mask features. The deposited features in each step are indicated by superimposed colored areas and arrows.

The three-angle metal deposition results in a threefold projection of all of the mask features with a spatial shift, except for the island, which is deposited only once. The axis of rotation [indicated by a dashed line in Fig. 4 (a)] is selected such 
that the island feature at $50^{\circ}$ tilting projects onto the side-wall of the top PMMA resist, and later on the deposited material is removed by lift-off.

In order to verify the spin-ratchet mechanism in Fig. 2, devices with ferromagnetic (F) leads made of CoFe that were used as spin detectors (FSF device) were fabricated. The spin polarization sign-change at $V=0$ is preserved, as when using normal leads, but the effective polarization of the leads, $P_{\mathrm{F}}$, is used to measure the relative contribution of cycles 01 and 21. For a quantitative measurement of the spin-ratchet efficiency, we independently determined $P_{\mathrm{F}}$. We accomplished this using similarly fabricated junctions embedded in nonlocal spin devices for which we obtained ${ }^{37} P_{\mathrm{F}} \approx$ 0.28 .

\subsection{Experimental set-up and characterization measurements (above $\Delta$ )}

Measurements were performed in a dilution refrigerator at $25 \mathrm{mK}$ with a true four-point ac/dc data acquisition technique. A dc voltage and a small superimposed ac sine voltage $(20 \mu \mathrm{V})$ were applied to the SET. Both the ac current component through the SET and the ac voltage across the normal leads were acquired using standard lock-in techniques. Therefore, the measurements both indicate true bias and conductance.

The electron transport properties of the SETs were fully characterized by means of differential conductance $\mathrm{d} I / \mathrm{d} V$ measurements at above-gap voltage bias from which we determined the device parameters, including the junctions' capacitances $C_{\mathrm{l}}$ and $C_{\mathrm{r}}$, the gate capacitance $C_{\mathrm{g}}$, and the superconducting gap $\Delta$. From the $\mathrm{d} I / \mathrm{d} V$ thresholds in Fig. 5 (FSF sample), the following parameters are obtained: $\Delta=303 \mu \mathrm{eV}, C_{1} \sim C_{\mathrm{r}} \approx 235 \mathrm{aF}, C_{\mathrm{g}} \approx 1.4 \mathrm{aF}, C_{\Sigma}=C_{1}+C_{\mathrm{r}}+C_{\mathrm{g}} \approx 470 \mathrm{aF}$, and $E_{\mathrm{c}}=e^{2} / 2 C_{\Sigma} \sim 170 \mu \mathrm{eV}$.

The resistances for the left and right junctions were estimated independently as $R_{1}=350 \mathrm{k} \Omega$ and $R_{\mathrm{r}}=70 \mathrm{k} \Omega$ from similarly fabricated isolated junctions and the total SET resistance $R_{1}+R_{\mathrm{r}}=420 \mathrm{k} \Omega$. Using these parameters, we obtained $\Gamma_{1}^{\mathrm{oe}} \approx 8 \times 10^{6} \mathrm{~s}^{-1}<\Gamma_{\mathrm{r}}^{\mathrm{oe}} \approx 4 \times 10^{7} \mathrm{~s}^{-1}<<\Gamma^{\mathrm{eo}} \approx 5 \times 10^{9} \mathrm{~s}^{-1}$ and $\alpha=\Gamma_{\mathrm{l}}^{\mathrm{oe}} / \Gamma_{\mathrm{r}}^{\mathrm{oe}} \approx R_{\mathrm{r}} / R_{\mathrm{l}} \approx 0.2$. Therefore, the device fulfills the rate hierarchy and should present spin ratchet effects.

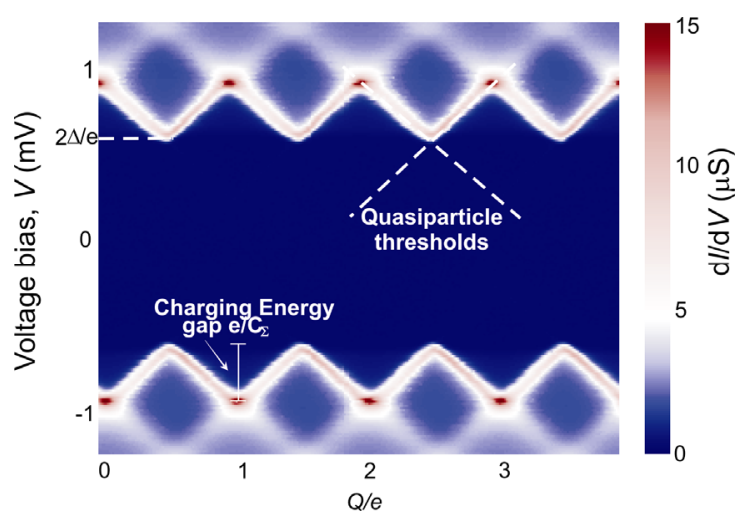

Figure 5. Experimental above-gap $\mathrm{d} I / \mathrm{d} V$ characteristics of a FSF device as a function of dc voltage $V$ across the SET and gate voltage $V_{\mathrm{g}}$. The $\mathrm{d} I / \mathrm{d} V$ amplitude is represented by a color scale from blue (zero) to red (15 $\left.\mu \mathrm{S}\right)$. From the voltage threshold for single quasiparticle events, the parameters $C_{1} \sim C_{\mathrm{r}} \approx 235 \mathrm{aF}, C_{\mathrm{g}} \approx 1.4 \mathrm{aF}$, and $\Delta \approx 303 \mu \mathrm{eV}$ are obtained. The lines are guides to the eye for the threshold voltages above the gap. $B=0, T=25 \mathrm{mK}$.

\section{EXPERIMENTAL RESULTS}

\subsection{Transport below $\Delta$, general trends}

Fig. 6 shows the evolution of $\mathrm{d} I / \mathrm{d} V$ as a function of the magnetic field at below-gap bias for the FSF device. At $B=0$, we observe a symmetric response about $V=0$ [Fig. 6 (a)]. There, $\mathrm{d} I / \mathrm{d} V$ is zero within the sensitivity of our measurements for voltage magnitudes below the gap, except at the quasiparticle thresholds, where it presents a peak whose intensity is nearly independent of $V$ and $V_{\mathrm{g}}$. This suggests that the Andreev cycle [Fig. 2 (b)] and cotunneling processes are suppressed. Integration of $\mathrm{d} I / \mathrm{d} V$ results in a current plateau $I_{\mathrm{p}} \sim 5.8 \mathrm{pA}$ beyond the thresholds. Because the 
current is limited by $\Gamma_{\mathrm{r}}^{\mathrm{oe}}, I_{\mathrm{p}} \sim e \Gamma_{\mathrm{r}}^{\mathrm{oe}} \sim 5 \mathrm{pA}$, which is in reasonable agreement with the measured value. This indicates that transport is dominated by tunneling events in the $r$ junction.

The below-gap quasiparticle thresholds cross at about $V_{0}=259 \mu \mathrm{V}$ [Fig. 6 (a)]. This is in agreement with $V_{0} \sim 2\left(\Delta-E_{\mathrm{c}}\right) / e$ [Fig. 2 (b)] when using $E_{\mathrm{c}}=170 \mu \mathrm{eV}$ and $\Delta \sim 303 \mu \mathrm{eV}$ as obtained from the above-gap thresholds (Fig. 5). At $B=1 \mathrm{~T}$, $V_{0}$ decreases to $94 \mu \mathrm{V}$ due to $E_{\mathrm{Z}}$. At $B=1.5 \mathrm{~T}, V_{0}$ becomes zero and the SET is in the pure spin ratchet regime [Fig. 2 (c)]. The decrease in $V_{0}$ with increasing $B$ is larger than expected if only $E_{\mathrm{Z}}$ is considered, in which case, the ratchet effect should occur at $B_{\mathrm{SR}} \approx 2.3 \mathrm{~T}$. This is due to a reduction of $\Delta$ by residual orbital depairing. When such a reduction is considered, $\Delta(1 \mathrm{~T})=272 \mu \mathrm{eV}, V_{0}(1 \mathrm{~T})=2\left[\Delta(1 \mathrm{~T})-E_{\mathrm{c}}\right] / e-E_{\mathrm{Z}} / e \approx 88 \mu \mathrm{V}$ is close to the measured value. Moreover, considering $\Delta(1.5 \mathrm{~T})=256 \mu \mathrm{eV}$, we estimate $\left.B_{\mathrm{SR}}=\Delta(1.5 \mathrm{~T})-E_{\mathrm{c}}\right] /\left(g \mu_{\mathrm{B}}\right) \approx 1.48 \mathrm{~T}$, in agreement with the observed result.

(a)

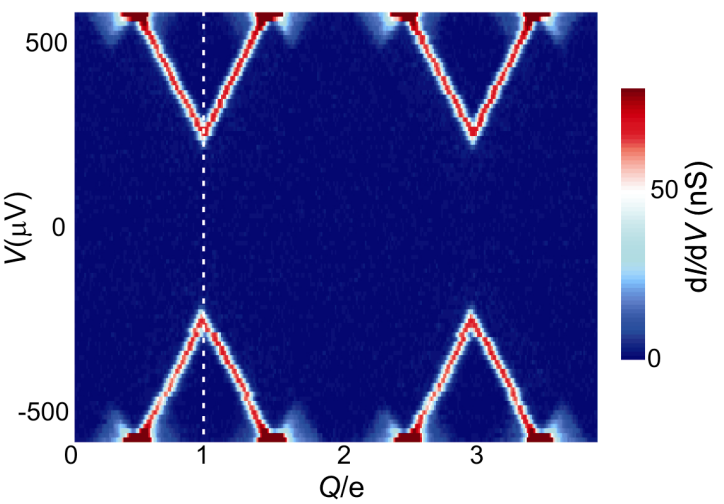

(c)

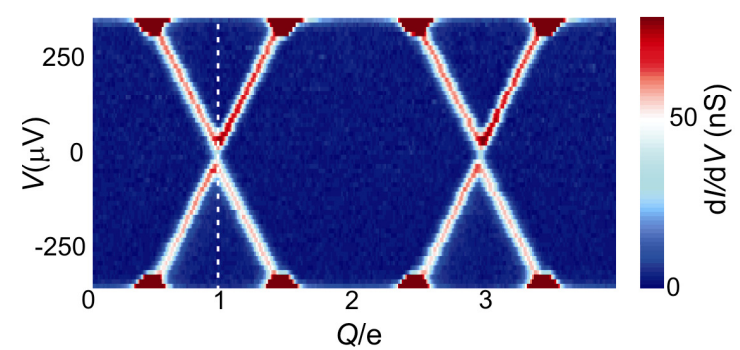

(b)

$B<B_{\mathrm{SR}}$

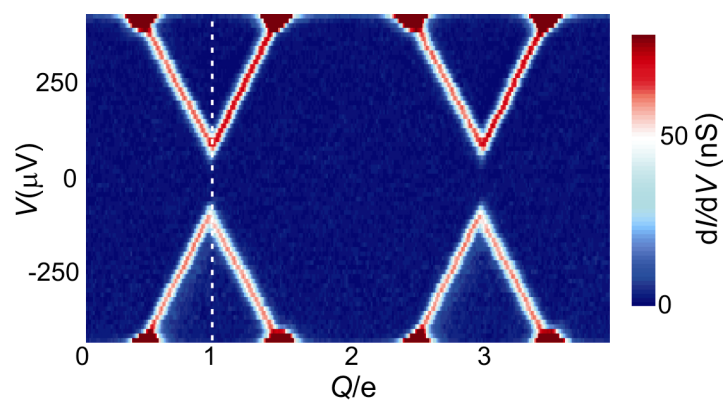

(d)

$B>B_{\mathrm{SR}}$

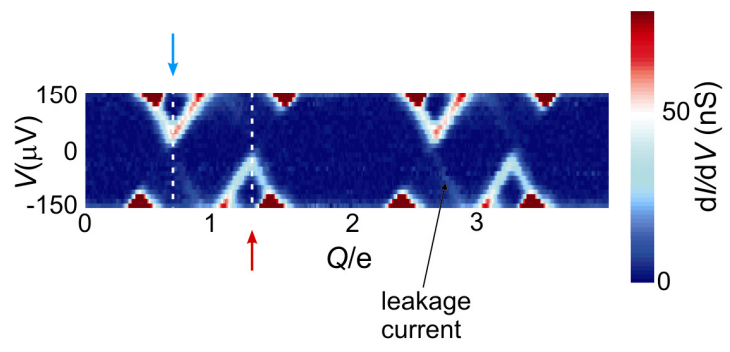

Figure 6. Below-gap transport in an applied magnetic field and characteristics of an SET spin ratchet. Differential conductance $\mathrm{d} I / \mathrm{d} V$ versus gate $V_{\mathrm{g}}$ and bias $V$ voltages. (a), $B=0$; (b), $B=1 \mathrm{~T}$; (c), $B=1.5 \mathrm{~T}$; (d), $B=2.5 \mathrm{~T}$.

\subsection{Bias voltage asymmetry and spin ratchet effect}

Of key importance, Fig. 7 (b) and (c) show that the differential conductance at $B \neq 0$ is no longer symmetric about $V=0$, presenting a larger magnitude for $V>0$ than for $V<0$ along the below-gap quasiparticle thresholds. This observation is consistent with the description in Fig. 2 and represents an experimental confirmation of the spin ratchet effect. Indeed, the asymmetry results from $P_{\mathrm{F}}$ and the fact that the current across the SET for positive and negative $V$ has opposite spin polarization. The leads are always magnetized parallel to each other along the $B$ direction and, because $P_{\mathrm{F}}>0$, they favor the dominant spin-down current cycle 01 at $V>0$ and hinder the dominant spin-up current cycle 21 at $V<0$.

We quantify the transport asymmetry using the parameter $\beta=\left(G_{\mathrm{p}}^{+}-G_{\mathrm{p}}^{-}\right) /\left(G_{\mathrm{p}}^{+}+G_{\mathrm{p}}^{-}\right)$, where $G_{\mathrm{p}}^{+}=\mathrm{d} I / \mathrm{d} V l_{\text {peak }}(V>0)$ and $G_{\mathrm{p}}^{-}=\mathrm{d} I / \mathrm{d} V l_{\text {peak }}(V<0)$ are the values of the peak conductances along the dotted white lines in Fig. 6 . At $B=0$ [Figs. 6 (a) and 7 (a)], $\beta$ is zero within the sensitivity of our measurements, as expected. At $B=1 \mathrm{~T}$ and $B=1.5 \mathrm{~T}$ [Fig. 6 (b) and (c), and Fig. 7, (b) and (c)], the difference between $G_{\mathrm{p}}^{+}$and $G_{\mathrm{p}}^{-}$becomes apparent resulting in $\beta \sim 0.14$ in both cases. 

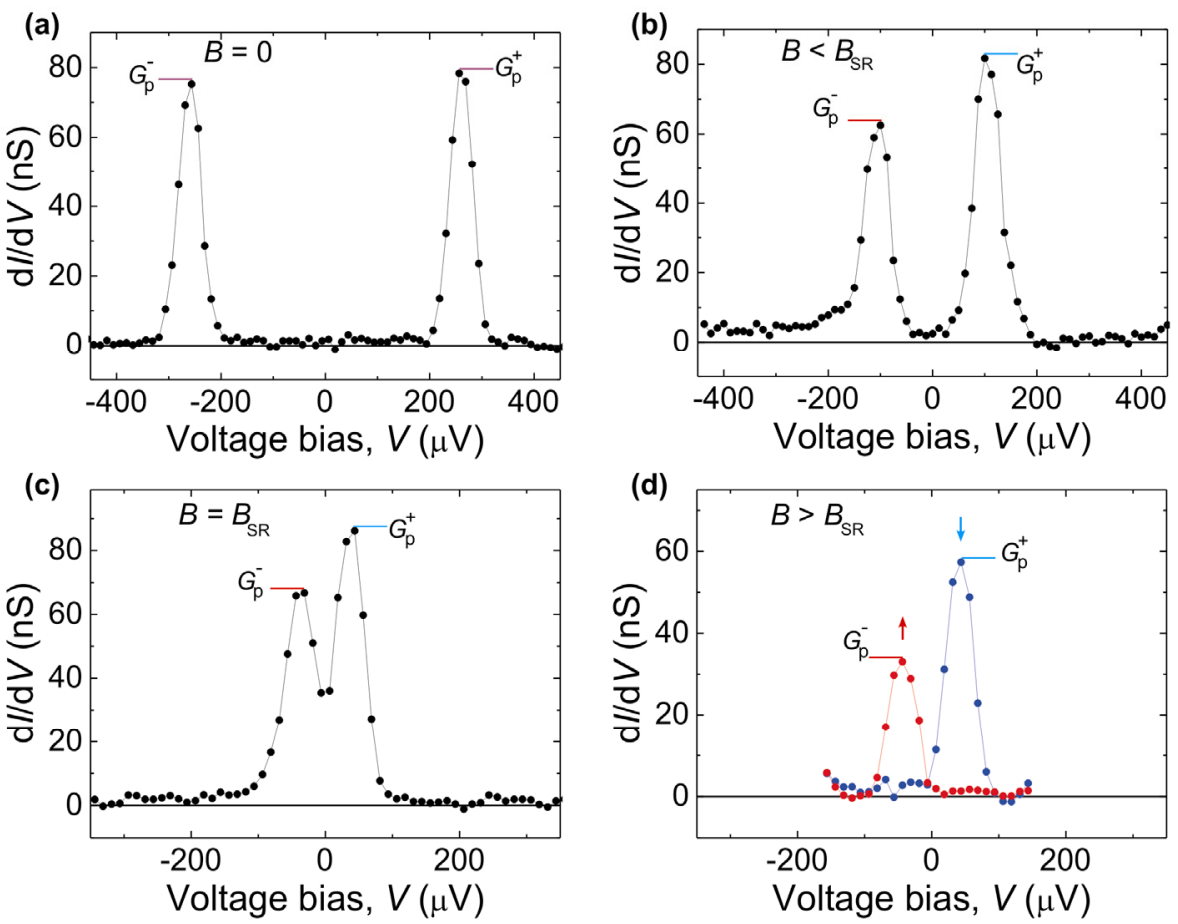

Figure 7. Spin filtering. (a), $B=0$; (b), $B=1 \mathrm{~T}$; (c), $B=1.5 \mathrm{~T}$; (d), $B=2.5 \mathrm{~T}$. Differential conductance $\mathrm{d} I / \mathrm{d} V$ versus $V$ cross-sections along the dotted white lines in Fig. 3. In (d), the red and blue curves are cross-sections along the white lines indicated with red and blue arrows in Fig. $6(\mathrm{~d})$, respectively.

We define the spin-ratchet efficiency $\eta$ as equal to the spin filtering capability $\eta \approx(1-\alpha) /(1+\alpha)$ of our device. For $\alpha \sim 0$, nearly perfect filtering, that is, $\eta \sim 1$, is achieved. In such scenario, $\beta$ directly measures the effective polarization of the leads; that is, $\beta=P_{\mathrm{F}}=0.28$. For $\alpha>0$, a decrease in filtering efficiency is expected and therefore $\beta$ should decrease accordingly as $\beta \approx \eta P_{\mathrm{F}}$. For our device $\alpha \sim 0.2$, we thus estimate $\eta \sim 0.67$ and $\beta \approx \eta P_{\mathrm{F}} \sim 0.19$, a value that is somewhat larger than that obtained with our measurements $(\beta \sim 0.14)$ which is equivalent to $\eta \sim 0.5$. This discrepancy could be related to the uncertainty in the estimation of $R_{\mathrm{l}, \mathrm{r}}$ or to Andreev reflections in one of the junctions, which could contribute an unpolarized component to the total current.

At magnetic fields $B>B_{\mathrm{SR}}$, where the spin-up and spin-down quasiparticle thresholds are resolved, the SET behaves as a diode that filters spin-up or spin-down quasiparticles [Figs. 6 (d) and 7 (d)]. Namely, the current should be fully spindown polarized for $V_{\mathrm{g}}$ about the degeneracy point (A) and spin-up polarized for $V_{\mathrm{g}}$ about the degeneracy point (B) in Fig. 2 (d). Accordingly, we calculate $\beta$ from the conductance peaks along the two dotted lines in Fig. 6 (d) obtaining $\beta \sim 0.26$, which is close to $P_{\mathrm{F}} \sim 0.28$ and indicates a filtering efficiency larger than 0.9 .

Lastly, we stress that the spin ratchet effect is related to quasiparticle tunneling through the high-transparency junction. To further show this, we fabricated devices with a normal $(\mathrm{N})$ metal lead made of $\mathrm{Cu}$ connected to the low-transparency junction (NSF). Here, $R_{1} \approx 650 \mathrm{k} \Omega$ and $R_{\mathrm{r}} \approx 70 \mathrm{k} \Omega$. As the high-transparency tunnel barrier connected to the ferromagnetic lead controls the transport, $\beta$ should remain close to $P_{\mathrm{F}}$, when calculated as in Fig. 7 (d). Moreover, because $R_{\mathrm{r}}$ in this device is estimated to be of the same order of magnitude as that of the FSF device, the conductance peaks should not be significantly affected. Both these observations agree with the experimental $\mathrm{d} I / \mathrm{d} V$ results shown in Fig. 8. At $B=0$ [Fig. 8 (a)], $\beta$ is again zero within the sensitivity of our measurements and, at $B>B_{\mathrm{SR}}$ [Fig. 8 (b)], $\beta \sim$ $0.25 \sim P_{\mathrm{F}}$, whereas the magnitudes of the conductance peaks compare well with those shown in Fig. 7. 


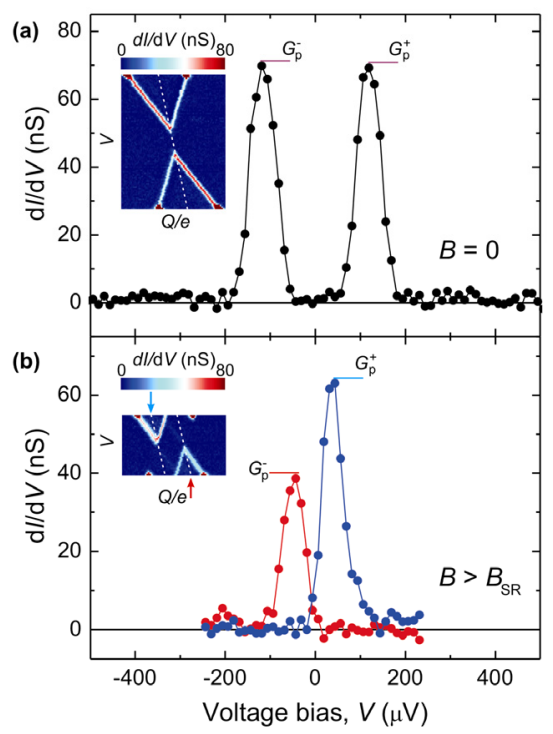

Figure 8. Spin filtering detection using an NSF sample. (a), $B=0 ;$ (b), $B=2 \mathrm{~T}$. The insets show $\mathrm{d} I / \mathrm{d} V$ versus gate $V_{\mathrm{g}}$ and bias $V$ voltages. The $\mathrm{d} I / \mathrm{d} V$ versus $V$ cross-sections (main panels) are taken along the corresponding dotted lines in the insets.

\section{CONCLUSIONS AND OUTLOOK}

We have proposed and demonstrated a spin ratchet at the single-electron level that shows a very high efficiency. The device relies on the ground state energetics of a (highly) asymmetric single electron transistor comprising a superconducting island and normal leads. Zeeman splitting favors the trapping of a quasiparticle in the island with a specific spin orientation and makes the (spin-down) quasiparticle state $(n=1)$ degenerate with both the zero $(n=0)$ and the one $(n=2)$ excess Cooper-pair states. Quasiparticles tunneling in or out the island is favored through the junction with the smallest tunnel resistance, mainly involving either the $n=0$ or the $n=2$ state in the transport. This results in spin polarized currents with opposite sign when the bias is reversed as required for the spin ratchet effect to be observed.

For our asymmetric single electron transistor $\alpha \sim 0.2$ for which we obtain a spin filter efficiency $\eta \sim 0.5$. For finite $\alpha$, small spin-down and spin-up leakage currents at negative and positive $V$, respectively, are expected. Such currents are deduced from weak conductance peaks in the diode with reverse bias [Fig. 7 (d)]. More efficient spin ratchets could be obtained in SETs designed with smaller $\alpha$, which could be achieved by incrementing the difference between $R_{\mathrm{l}}$ and $R_{\mathrm{r}}$. For $\alpha \sim 0.1, \eta$ would exceed 0.8 and for $\alpha \sim 0.05$, it would exceed 0.9. Such values of $\alpha$, which require a small transparency in one of the junctions, are possible without a decrease in the overall current through the SET because transport is dominated by the tunneling rate $\Gamma_{\mathrm{r}}^{\mathrm{oe}}$ in the transparent junction $r$.

This research paves the way for a new means to study spin-related phenomena. Because the spin ratchets presented here work at the single-electron level, they can, for example, be used to initialize and readout the state of spin-based quantum bits or to identify the spin orientation of single electrons in a test of the Einstein-Podolsky-Rosen paradox with spinentangled electrons.

\section{ACKNOWLEDGEMENTS}

We gratefully acknowledge support from M. Tinkham. Samples were fabricated at the Center for Nanoscale Systems (CNS), Harvard University. This research was supported by the Spanish Ministerio de Ciencia e Innovación, MICINN (MAT2010-18065, FIS2009-06671-E). 


\section{REFERENCES}

[1] Žutić, I., Fabian, J. and Das Sarma, S., "Spintronics: fundamentals and applications," Rev. Mod. Phys. 76, 323 (2004).

[2] Awschalom, D. D., Loss, D. and Samarth, N. [Semiconductor Spintronics and Quantum Computation], Springer-Verlag, Berlin, (2002).

[3] Recher, P., Sukhorukov, E. V. and Loss, D. ,QQuantum dot as spin filter and spin memory,” Phys. Rev. Lett. 85, 1962-1965 (2000).

[4] Hanson, R. et al., "Semiconductor few-electron quantum dot operated as a bipolar spin filter," Phys. Rev. B 70, 241304(R) (2004).

[5] Merchant, C. A. and Marković, N., "Electrically tunable spin polarization in a carbon nanotube spin diode" Phys. Rev. Lett. 100, 156601 (2008)

[6] Sharma, P. and Chamon, C., "Quantum pump for spin and charge transport in a Luttinger liquid," Phys. Rev. Lett. 87, 096401(2001).

[7] Mucciolo, E. R., Chamon, C. and Marcus, C. M. "Adiabatic quantum pump of spin-polarized current," Phys. Rev. Lett. 89, 146802 (2002).

[8] Watson, S. K., Potok, R. M., Marcus, C. M. and Umansky, V., "Experimental realization of a quantum spin pump," Phys. Rev. Lett. 91, 258301 (2003).

[9] Scheid, M., Bercioux, D. and Richter, K., "Zeeman ratchets: pure spin current generation in mesoscopic conductors with non-uniform magnetic fields," New J. Phys. 9, 401 (2007).

[10] Smirnov, S., Bercioux, D., Grifoni, M. and Richter, K., "Quantum dissipative Rashba spin ratchets," Phys. Rev. Lett. 100, 230601 (2008).

[11]Flatté, M. E., "Spin ratchets: A one-way street for spin current," Nature Phys. 4, 587-588 (2008).

[12] Scheid, M., Lassl, A. and Richter, K., "Resonant-tunneling-based spin ratchets," EPL 87, 17001 (2009).

[13] Hänggi, P., Marchesoni, F. and Nori, F., "Brownian motors," Ann. Phys. 14, 51 (2005).

[14] Hänggi, P. and Marchesoni, F., "Artificial Brownian motors: Controlling transport on the nanoscale," Rev. Mod. Phys. 81, 387-442 (2009).

[15] Song, A. M., Lorke, A., Kriele, A., Kotthaus, J. P. Wegscheider, W. and Bichler M., "Nonlinear electron transport in an asymmetric microjunction: a ballistic rectifier," Phys. Rev. Lett. 80, 3831-3834 (1998).

[16] Linke, H. et al., "Experimental tunneling ratchets," Science 286, 2314 (1999).

[17] Valenzuela, S. O., "Order and mobility of solid vortex matter in oscillatory driving currents," Phys. Rev. Lett. 88, 247003 (2002).

[18]Villegas J. E., Savel'ev S., Nori F., Gonzalez E. M., Anguita J. V., Garcia R., and Vicent J. L., "A superconducting reversible rectifier that controls the motion of magnetic flux quanta," Science 302, 1188-1191 (2003).

[19] Silva, C. C. D. et al., "Controlled multiple reversal of a ratchet effect," Nature 440, 651-654 (2006).

[20] Togawa, Y. et al., "Direct bbservation of rectified motion of vortices in a niobium superconductor," Phys. Rev. Lett. 95, 087002 (2005).

[21] Majer, J. B. et al., "Quantum ratchet effect for vortices,” Phys. Rev. Lett. 90, 056802 (2003).

[22]Zapata, I., Bartussek, R., Sols, F. and P. Hänggi, P., "Voltage rectification by a SQUID ratchet," Phys. Rev. Lett. 77, 2292-2295 (1996).

[23] Costache, M. V. and Valenzuela, S. O., "Experimental spin ratchet," Science 330, 1645-1648 (2010).

[24] Averin, D.V. and Nazarov, Yu. V., "Single-electron charging of a superconducting island," Phys. Rev. Lett. 69, 1993-1996 (1992).

[25] Tuominen, M. T., Hergenrother, J. M., Tighe, T. S. and Tinkham, M., "Experimental evidence for parity-based 2e periodicity in a superconducting single-electron tunneling transistor," Phys. Rev. Lett. 69, 1997-2000 (1992).

[26]Eiles, T.M., Martinis, J. M. and Devoret, M. H., "Even-odd asymmetry of a superconductor revealed by the Coulomb blockade of Andreev reflection," Phys. Rev. Lett. 70, 1862-1865 (1993).

[27] Hekking, F. W. J., Glazman, L. I., Matveev, K. A. and Shekhter, R. I., "Coulomb blockade of two-electron tunneling," Phys. Rev. Lett.70, 4138-4141 (1993).

[28] Schön, G. [Quantum transport and dissipation], Eds. Dittrich et al. T. Wiley-VCH Verlag GmbH, Weinheim, Ch 3 (1998).

[29] Hergenrother, J. M., Tuominen, M. T. and Tinkham, M., "Charge transport by Andreev reflection through a mesoscopic superconducting island," Phys. Rev. Lett. 72, 1742 (1994). 
[30] van Wees, B. J., de Vries, P., Magnée, P. and Klapwijk, T. M., "Excess conductance of superconductorsemiconductor interfaces due to phase conjugation between electrons and holes," Phys. Rev. Lett. 69, 510 (1992).

[31] Hekking, F. W. J. and Nazarov, Yu. V., "Interference of two electrons entering a superconductor," Phys. Rev. Lett. 71, 1625 (1993).

[32] Zaikin, A. D., "Influence of Coulomb and proximity effects on electron tunneling through normal metalsuperconductor interfaces," Physica B 203, 255 (1994).

[33] Žutić, I. and Valls, O. T., "Tunneling spectroscopy for ferromagnet/superconductor junctions,” Phys. Rev. B 61, 1555 (2000).

[34] Ferguson, A. J., Andresen, S. E., Brenner, R. and Clark, R. G., "Spin-dependent quasiparticle transport in aluminum single-electron transistors," Phys. Rev. Lett. 97, 086602 (2006).

[35] Valenzuela, S. O. and Tinkham, M., "Electrical detection of spin currents: The spin-current induced Hall effect (invited)," J. Appl. Phys. 101, 09B103 (2007).

[36] Valenzuela, S. O. and Tinkham, M., "Spin-polarized tunneling in room-temperature mesoscopic spin valves" Appl. Phys. Lett. 85, 5914-5916 (2007).

[37] Valenzuela, S. O., "Nonlocal spin detection, spin accumulation and the spin Hall effect," Int. J. Mod. Phys. B 23, 2413-2438 (2009). 